\title{
Atypical Imaging Features of Epstein-Barr Virus-Positive Primary Central Nervous System Lymphomas in Patients without AIDS
}

\author{
H.Y. Lee, H.S. Kim, J.W. Park, H.J. Baek, S.J. Kim, and C.-G. Choi
}

\begin{abstract}
BACKGROUND AND PURPOSE: Recent clinical experience with EBV-positive PCNSL in patients without acquired immune deficiency syndrome showed that they tended to have atypical features seen on conventional MR imaging. The purpose of our study was to evaluate the MR imaging features of EBV-positive PCNSL in patients without AIDS and to compare these imaging findings with those of EBV-negative PCNSL.
\end{abstract}

MATERIALS AND METHODS: MR images were obtained in 55 consecutive patients with pathologically proved EBV-positive ( $n=10$ ) or EBV-negative $(n=45)$ PCNSL. We statistically analyzed the differences between the patient groups regarding the occurrence of tumor necrosis or hemorrhage and $\mathrm{ADC}, \mathrm{rCBV}_{\text {max }}, \mathrm{rCBV}_{\mathrm{r}}$, and the Cho/NAA ratio in the tumor area.

RESULTS: Tumor necrosis and hemorrhage were observed in $9(90 \%)$ and $7(70 \%)$, respectively, of the patients with EBV-positive PCNSL; necrosis was observed in $8(18 \%)$, and hemorrhage, in $3(7 \%)$ patients with EBV-negative PCNSL $(P<.0001$ each). The necrotic core was hyperintense relative to contralateral white matter, as seen on DWI in 4 patients with EBV-positive PCNSL, though the ADC between the 2 patient groups did not differ significantly. $\mathrm{rCBV}_{\text {max }}, \mathrm{rCBV}_{\mathrm{r}}$, and the Cho/NAA ratios did not differ significantly between the 2 groups. The sensitivity and specificity of necrosis and hemorrhage for differentiating the 2 groups were $89.2 \%$ and $81.7 \%$ and $78.5 \%$ and $94.1 \%$, respectively.

CONCLUSIONS: Our initial clinical experience with a small number of patients suggests that EBV-positive PCNSL in patients without AIDS tends to present with atypical MR imaging features.

ABBREVIATIONS: DSC = dynamic susceptibility-weighted contrast-enhanced; EBV = Epstein-Barr virus; PCNSL = primary central nervous system lymphoma; $\mathrm{rCBV}_{\max }=$ maximum value of relative cerebral blood volume; $r C B V_{r}=$ range of relative cerebral blood volume

$\mathrm{E}^{\mathrm{B}}$

BV has been implicated in the pathogenesis of PCNSL in patients with congenital and acquired immunodeficiency, ${ }^{1,2}$ though not in immunocompetent patients. Nevertheless, EBV has been observed in PCNSL in immunocompetent hosts, thus suggesting its role in PCNSL tumorigenesis in these individuals. ${ }^{1-3}$

Age-related, EBV-positive, diffuse, large B-cell lymphoma is a new disease entity in the 2008 World Health Organization classification occurring in the elderly ${ }^{4,5}$ and mostly without any known immunodeficiency or prior lymphoma. This new disease entity has been characterized as having a propensity to involve extra-

Received July 23, 2012; accepted after revision October 11.

From the Department of Radiology (H.Y.L., H.S.K., J.W.P., S.J.K., C.-G.C.), Research Institute of Radiology, Asan Medical Center, University of Ulsan College of Medicine, Seoul, Korea; Department of Radiology (H.J.B.), Haeundae Paik Hospital, Inje University College of Medicine, Busan, South Korea; and Department of Radiology (H.Y.L.), Inha University Hospital, Inha University School of Medicine, Incheon, Korea.

Please address correspondence to Ho Sung Kim, MD, Department of Radiology, Research Institute of Radiology, Asan Medical Center, University of Ulsan, College of Medicine, 86, Asanbyeongwon-gil,Songpa-gu, Seoul, 138-736, Korea; e-mail: radhskim@gmail.com

http://dx.doi.org/10.3174/ajnr.A3429 nodal sites and to be associated with a broad morphologic spectrum ranging from the precursor-reactive proliferation of lymphoid cells to overt lymphoma. ${ }^{6-9}$

To our knowledge, there have been no radiologic reports of CNS involvement of EBV-associated diffuse large-B-cell lymphoma in patients without acquired immune deficiency syndrome. We have encountered 10 consecutive patients without AIDS with EBV-positive PCNSL since June 2008. We hypothesized that EBV-positive PCNSL may show imaging features that differ from those of EBV-negative PCNSL but may have imaging features similar to those of PCNSL in patients with AIDS. We, therefore, assessed 10 consecutive patients without AIDS with EBV-positive PCNSL by using both conventional and advanced MR imaging and compared these imaging findings with those of patients without AIDS with EBV-negative PCNSL.

\section{MATERIALS AND METHODS Study Population}

This retrospective study was approved by our institutional review board. One hundred twenty-seven consecutive patients were identified from the data base of our institution as having pathologically proved PCNSL between January 2004 and May 2012. Of 
Table 1: Demographic data of the 10 patients with EBV-positive PCNSLs

\begin{tabular}{|c|c|c|c|c|c|c|c|c|}
\hline $\begin{array}{l}\text { Patient } \\
\text { No. }\end{array}$ & $\begin{array}{l}\text { Age } \\
(y r) / \\
\text { Sex }\end{array}$ & Clinical Symptom & $\begin{array}{l}\text { Imaging } \\
\text { Diagnosis }\end{array}$ & Surgery & Pathologic Diagnosis & $\begin{array}{l}\text { Treatment } \\
\text { Response }\end{array}$ & $\begin{array}{c}\text { Follow-Up } \\
\text { Periods } \\
\text { (mo) }\end{array}$ & $\begin{array}{l}\text { Follow-Up } \\
\text { Results }\end{array}$ \\
\hline 1 & $34 / \mathrm{M}$ & Dizziness & Metastasis & Resection & Diffuse large B-cell lymphoma & PD & 9 & Death \\
\hline 2 & $55 / \mathrm{M}$ & Left weakness & Lymphoma & Biopsy & Diffuse large B-cell lymphoma & CR & 15 & Alive \\
\hline 3 & $62 / M$ & Left weakness & Glioblastoma & Resection & Diffuse large B-cell lymphoma & $\mathrm{CR}$ & 30 & Alive \\
\hline 4 & $70 / \mathrm{M}$ & Left weakness & Glioblastoma & Resection & Diffuse large B-cell lymphoma & $\mathrm{CR}$ & 13 & Alive \\
\hline 5 & $83 / \mathrm{M}$ & Dysarthria & Glioblastoma & Resection & Diffuse large B-cell lymphoma & Loss & 17 & Lost to $\mathrm{F} / \mathrm{U}$ \\
\hline 6 & $62 / \mathrm{M}$ & Dysarthria & Glioblastoma & Resection & Diffuse large B-cell lymphoma & PR & 3 & Alive \\
\hline 7 & $60 / \mathrm{M}$ & Dizziness & Lymphoma & Biopsy & Diffuse large B-cell lymphoma & PR & 50 & Alive \\
\hline 8 & $76 / F$ & $\begin{array}{l}\text { Weakness in both } \\
\text { hands }\end{array}$ & Lymphoma & Biopsy & Diffuse large B-cell lymphoma & PR & 27 & Death \\
\hline 9 & $29 / \mathrm{F}$ & Left hemiparesis & Fungal infection & Biopsy & Diffuse large B-cell lymphoma & PR & 33 & Alive \\
\hline 10 & $59 / \mathrm{F}$ & Right weakness & Glioblastoma & Resection & Diffuse large B-cell lymphoma & PR & 3 & Alive \\
\hline
\end{tabular}

Note:-CR indicates complete remission; PR, partial treatment response; PD, progression of disease; F/U, follow-up.

these 127 patients, we excluded 70 without EBV-encoded RNA in situ hybridization. PCNSL in patients considered immunocompetent and not associated with HIV has infrequently been demonstrated to be positive for EBV by using in situ hybridization techniques. Therefore, tumor tissue from PCNSL in patients considered immunocompetent has seldom been routinely tested for EBV at our institution when a biopsy is performed. The initial conventional and advanced MR imaging findings typical for high-grade gliomas or PCNSLs in immunocompromised patients prompted some clinical confusion before biopsy confirmation of the diagnosis. We performed EBV-encoded RNA in situ hybridization studies of the lymphoma tissue. Among the 57 patients with adequate EBV-encoded RNA in situ hybridization results, EBV was positive in 12 patients. Human immunodeficiency virus was identified in 2 patients with EBV-positive PCNSL. After we excluded 2 patients with human immunodeficiency virus infection, 55 consecutive patients without AIDS (10 with EBV-positive PCNSL, 45 with EBV-negative PCNSL) were finally enrolled in our study. Among the $10 \mathrm{EBV}$-positive patients with PCNSL, there were 7 men and 3 women (mean age, 59 years; age range, $29-83$ years). There were 30 men and 15 women (mean age, 57 years; age range, 13-76 years) among the 45 EBV-negative patients with PCNSL. A complete set of conventional brain MR imaging scans was obtained in all 55 study patients Among these patients, 29 (9 with EBV-positive, 20 with EBV-negative PCNSL) underwent advanced MR imaging, including DWI, DSC perfusion MR imaging, and MR spectroscopy. The demographic data of the 10 patients with EBV-positive PCNSL are found in Table 1.

\section{MR Imaging Protocol}

MR imaging was performed by using a 3T system (Achieva; Philips Healthcare, Best, the Netherlands) with an 8-channel sensitivity-encoding head coil. Our MR imaging protocol included transverse T2- and T1-weighted fast spin-echo imaging; transverse FLAIR spin-echo imaging; susceptibility-weighted imaging; diffusion-weighted spin-echo imaging; DSC perfusion MR imaging; contrast-enhanced T1-weighted 3D gradient-echo imaging, with transverse, coronal, and sagittal planes; and MR spectroscopy. Transverse, T2-weighted fast spin-echo images were obtained with the following parameters: TR/TE. 3000/80 ms; FOV, $23 \mathrm{~cm}$; section thickness, $5 \mathrm{~mm}$; matrix, $512 \times 512$; and acquisition time, 1 minute 48 seconds. Transverse T1-weighted fast spinecho images were obtained with the following parameters: TR/TE,
500/10 ms; FOV, $23 \mathrm{~cm}$; section thickness, $5 \mathrm{~mm}$; matrix, $512 \times$ 512; and acquisition time, 3 minutes 6 seconds. FLAIR spin-echo images were obtained with the following parameters: TR/TE, 11,000/125 ms; FOV, $23 \mathrm{~cm}$; section thickness, $5 \mathrm{~mm}$; matrix, $512 \times 512$; and acquisition time, 2 minutes 56 seconds. The SWI data were acquired by using a $3 \mathrm{D}$ flow-compensated gradient recalled-echo sequence with the following parameters: TR/TE, 24/34 ms; flip angle, $10^{\circ}$; FOV, $20 \mathrm{~cm}$; matrix, $332 \times 332$; section thickness, $3 \mathrm{~mm}$; slab thickness, $135 \mathrm{~mm}$; and total acquisition time, 4 minutes 2 seconds.

DWI was obtained with the following parameters: TR/TE, $3000 / 56 \mathrm{~ms}$; diffusion gradient encoding, $b=0,1000 \mathrm{~s} / \mathrm{mm}^{2}$; FOV, $25 \mathrm{~cm}$; section thickness, $5 \mathrm{~mm}$; matrix, $256 \times 256$; and acquisition time, 39 seconds. Contrast-enhanced transverse, coronal, and sagittal T1-weighted 3D gradient-echo images were obtained with the following parameters: TR/TE, 9.8/4.6 ms; FOV, $22.4 \mathrm{~cm}$; section thickness, $3 \mathrm{~mm}$; matrix, $448 \times 448$; and acquisition time, 5 minutes 56 seconds. Contrast-enhanced T1weighted images were obtained after intravenous administration of $0.1 \mathrm{mmol} / \mathrm{kg}$ of gadoterate meglumine (Gd-DOTA, Dotarem; Guerbet, Roissy Charles-de-Gaulle, France). DSC perfusion MR imaging was performed with gradient-echo-planar sequences during the administration of a standard dose of $0.1 \mathrm{mmol} / \mathrm{kg}$ of gadoterate meglumine (Dotarem) at a rate of $4 \mathrm{~mL} / \mathrm{s}$ by using an MR imaging-compatible power injector (Spectris MR injector; MedRad, Indianola, Pennsylvania). The parameters of DSC perfusion MR imaging were as follows: TR/TE, 1778/40 ms; flip angle, $35^{\circ}$; FOV, 20; section thickness, $5 \mathrm{~mm}$; matrix, $128 \times 128$; and acquisition time, 1 minute 53 seconds. We used a point-resolved spectroscopy sequence-based single-voxel proton MR spectroscopy with an intermediate TE of $144 \mathrm{~ms}$, a TR of $2000 \mathrm{~ms}$, and a flip angle of $90^{\circ}$.

\section{Image Analyses}

Two neuroradiologists (H.S.K. and H. Y. L., with 8 and 3 years of clinical experience, respectively) retrospectively reviewed all of the MR images. The lesion characteristics analyzed on conventional MR imaging included the number, anatomic location, shape, necrosis, and hemorrhage. Necrotic components were differentiated as seen on contrast-enhanced T1-weighted images as the interior of the enhanced lesions. Intratumoral macrohemorrhages were identified when there were high-signal-intensity intratumoral lesions seen on unenhanced T1-weighted MR images 
Table 2: Comparison of clinical and MR imaging findings between patients with EBV-positive PCNSL and those with EBV-negative PCNSL

\begin{tabular}{|c|c|c|c|c|c|}
\hline & EBV $(+)$ & No. & EBV (-) & No. & $P$ Value \\
\hline \multicolumn{6}{|l|}{ Clinical parameters } \\
\hline Age (mean) (yr) & 57.8 & 10 & 57.1 & 45 & .872 \\
\hline Sex (male/female) & $7 / 3$ & 10 & $30 / 15$ & 45 & .725 \\
\hline Follow-up periods (wk) & $3-50$ & 10 & $2-143$ & 45 & N/A \\
\hline Median progression (wk) & N/A & 10 & 58 & 45 & N/A \\
\hline \multicolumn{6}{|l|}{ Conventional imaging } \\
\hline Multiplicity (single/multiple) & $6 / 4$ & 10 & $23 / 22$ & 45 & .715 \\
\hline Location (PV/lobar/both) & $1 / 6 / 3$ & 10 & 22/12/11 & 45 & .122 \\
\hline Shape (irregular/lobulating) & $2 / 8$ & 10 & $27 / 18$ & 45 & .067 \\
\hline Necrosis $(+/-)$ & $9 / 1$ & 10 & $8 / 37$ & 45 & .0001 \\
\hline Hemorrhage $(+/-)$ & $7 / 3$ & 10 & $3 / 42$ & 45 & $<.0001$ \\
\hline \multicolumn{6}{|l|}{ Advanced imaging } \\
\hline $\mathrm{ADC}_{\text {solid }}$ (mean) & $(0.77 \pm 0.15) \times 10^{-3} \mathrm{~mm}^{2} / \mathrm{s}$ & 8 & $(0.74 \pm 0.27) \times 10^{-3} \mathrm{~mm}^{2} / \mathrm{s}$ & 20 & .761 \\
\hline $\mathrm{rCBV}_{\max }($ mean) & $2.69 \pm 0.79$ & 9 & $2.65 \pm 0.81$ & 20 & .715 \\
\hline $\mathrm{rCBV}_{\mathrm{r}}$ (mean) & $1.82 \pm 0.72$ & 9 & $1.75 \pm 0.86$ & 20 & .729 \\
\hline Cho/NAA ratio & $6.20 \pm 8.28$ & 6 & $8.11 \pm 7.22$ & 16 & .269 \\
\hline
\end{tabular}

Note:- + indicates positive; -, negative; N/A, not applicable; PV, periventricular.

and low- or dark-signal-intensity lesions seen on T2-weighted fast-spin-echo images and/or as seen on conventional gradientecho images as areas of low signal intensity. High-resolution susceptibility-weighted imaging was optionally performed to confirm the equivocal findings of hemorrhage. The lesions were classified as single focal or countable multifocal or innumerable disseminated lesions; according to anatomic location, including periventricular, lobar, or periventricular with lobar; according to their irregular, round, or lobulating shape; and according to the occurrence of necrosis or hemorrhage. Two readers blinded to the clinical and histopathologic findings performed qualitative visual inspection of both the conventional and the advanced MR images of PCNSL with a consensus reading.

Regarding the analysis of DWI and ADC maps, the region of interest was drawn in solid portions of the tumors $\left(\mathrm{ADC}_{\text {solid }}\right)$, and the $\mathrm{ADC}$ value of each was measured by using image-analysis software (Diffusion Lab TM; Clinical Imaging Solution, Seoul, South Korea). Parametric maps were obtained from the DSC perfusion MR imaging results by using a dedicated software package (nordicICE; NordicImagingLab, Bergen, Norway) and in-house software by using Matlab 2010b (MathWorks, Natick, Massachusetts) for semiquantitative perfusion analysis. On a pixel-by-pixel basis, regional CBV maps were normalized by dividing each regional CBV value in a specific section by that of unaffected white matter, as defined by an experienced neuroradiologist (H.S.K., with 8 years of clinical experience). We manually drew $2.5-\mathrm{mm}$ diameter circular ROIs onto each enhancing mass in a selected representative imaging section of a coregistered normalized CBV map, with the size of the region of interest being kept constant. Histograms were generated by classification of the normalized CBVs in each region of interest, and the interval between the minimum and maximum pixel values was divided into 200 equally spaced bins. ${ }^{10,11}$ The range of normalized CBVs along the $\mathrm{X}$-axis was kept constant (between zero and 10). We recorded the $\mathrm{rCBV}_{\text {max }}$ and $\mathrm{rCBV}_{\mathrm{r}}$. MR spectra were analyzed by using the manufacturer's spectroscopy software package (SpectroView, Philips Healthcare). The Cho/NAA ratio was calculated and used for analysis. For quantitative analysis of advanced MR imaging, the measurements of parametric maps, including ADC and $\mathrm{rCBV}$, were approved by a neuroradiologist (H.S.K.) experienced with diffusion and perfusion data acquisitions at our institution and once again blinded to the conventional imaging findings.

\section{Statistical Analyses}

Differences in the clinical and MR imaging features were compared in patients with EBV-positive and -negative PCNSL by using nonparametric statistics, including the Fisher exact test or the Mann-Whitney $U$ test, as appropriate. The performance of conventional MR imaging parameters was evaluated with sensitivity and specificity. All statistical analyses were performed by using the Statistical Package for the Social Sciences software (Version 19.0 for Windows; SPSS, Chicago, Illinois), and statistical significance was defined as $P<.05$.

\section{RESULTS}

\section{Conventional Imaging Findings}

Of the 10 study patients with EBV-positive PCNSL, multiple lesions were observed in 4 patients (40\%). Lobar location was the most frequent ( 6 patients, $60 \%$ ), followed by periventricular with lobar $(3,30 \%)$ and periventricular $(1,10 \%)$ locations. The lesions were round or lobulated in 8 patients $(80 \%)$ and irregularly shaped in $2(20 \%)$.

Of the 45 patients with EBV-negative PCNSL, there were multiple lesions in 22 patients (49\%). The lesion location was periventricular in $22(49 \%)$, lobar in $12(27 \%)$, and periventricular with lobar in 11 patients (24\%). The shape of the lesion was round or lobulating in 18 patients (40\%) and irregular in 27 (60\%).

Hemorrhage was observed in 7 (70\%) and necrosis, in $9(90 \%)$ of the EBV-positive patients, while hemorrhage was observed in 3 $(7 \%)$ and necrosis, in 8 (18\%) EBV-negative patients. Tumor necrosis and hemorrhage differed significantly in patients with EBV-positive and -negative PCNSL $(P<.0001$, each) (Table 2$)$. The sensitivity and specificity for multiplicity were $55.6 \%$ and $53.5 \%$; for location, $88.9 \%$ and $48.8 \%$; for shape of the lesion, $77.8 \%$ and $58.1 \%$; for tumor necrosis, $89.2 \%$ and $81.7 \%$; and for hemorrhage, $78.5 \%$ and $94.1 \%$. Unusually rapid lesion progression was observed in a 62-year-old man (Fig 1). Initially, the lesion in this patient was solitary and showed thin rim enhancement. However, the lesion grew in size, with intratumoral hemorrhage 

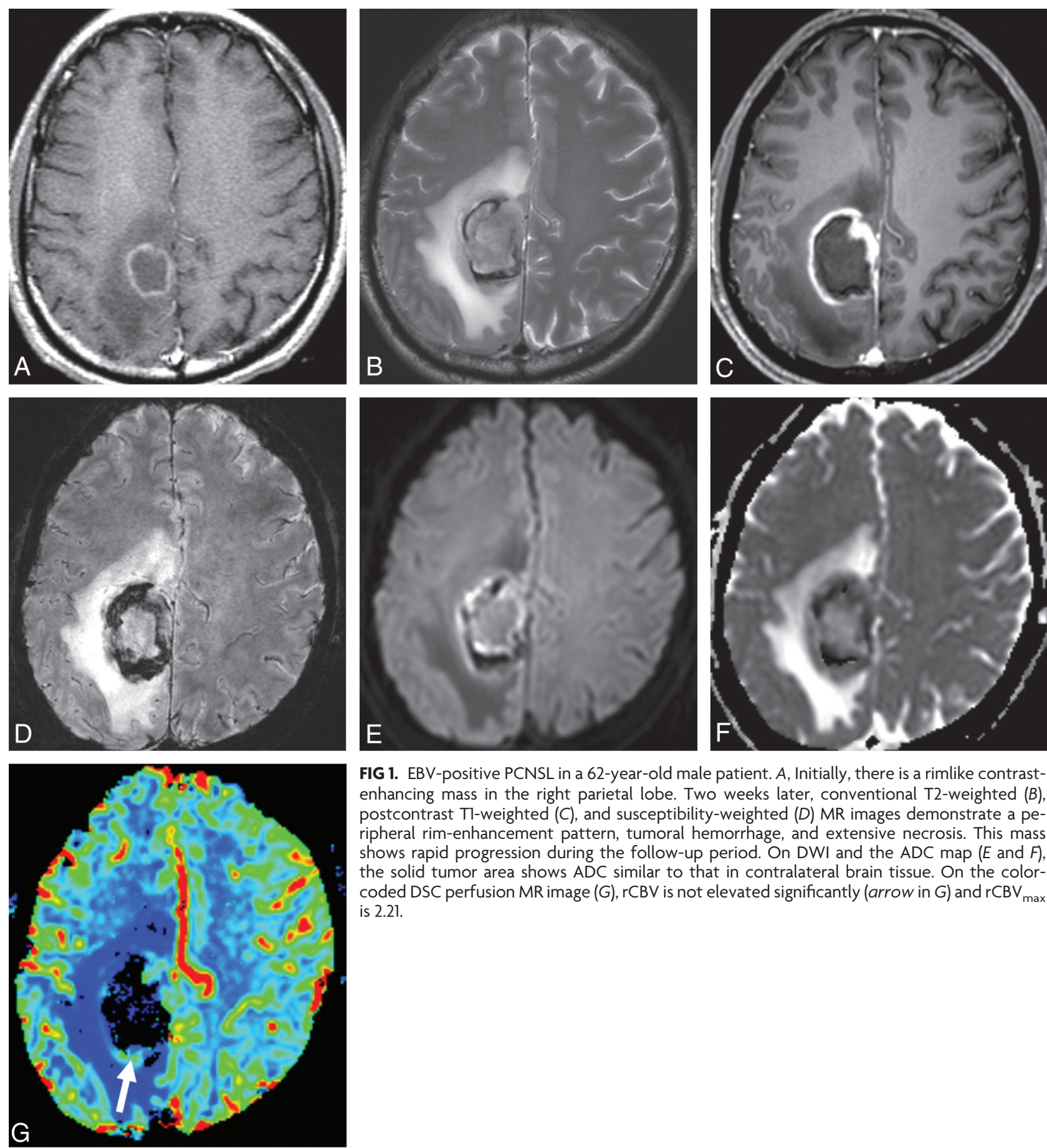

FIG 1. EBV-positive PCNSL in a 62 -year-old male patient. $A$, Initially, there is a rimlike contrastenhancing mass in the right parietal lobe. Two weeks later, conventional T2-weighted $(B)$, postcontrast T1-weighted $(C)$, and susceptibility-weighted $(D)$ MR images demonstrate a peripheral rim-enhancement pattern, tumoral hemorrhage, and extensive necrosis. This mass shows rapid progression during the follow-up period. On DWI and the ADC map ( $E$ and $F$ ), the solid tumor area shows ADC similar to that in contralateral brain tissue. On the colorcoded DSC perfusion MR image (G), rCBV is not elevated significantly (arrow in $G$ ) and rCBV max $_{\text {max }}$ is 2.21 .

and irregularly enhancing portions becoming evident on follow-up MR imaging performed 11 days later.

\section{Advanced Imaging Findings}

Nine patients with EBV-positive and 20 with EBV-negative PCNSL were assessed by using advanced MR imaging, including DWI, DSC perfusion MR imaging, and MR spectroscopy. All enhancing tumors in these 29 patients showed highly cellular areas on DWI and ADC (Figs. 1 and 2). One patient with EBV-positive PCNSL had extensive subacute hemorrhage, and the ADC map was not informative due to the susceptibility artifacts. Of the remaining 8 patients with necrotic EBV-positive PCNSL, the mean for $\mathrm{ADC}_{\text {solid }}$ was $0.77 \times 10^{-3}$ $\mathrm{mm}^{2} / \mathrm{s}$. The mean for $\mathrm{ADC}_{\text {solid }}$ was $0.74 \times 10^{-3} \mathrm{~mm}^{2} / \mathrm{s}$ in 20 patients with necrotic EBV-negative PCNSL. All the enhancing lesions in 9 cases of EBV-positive and 20 cases of EBV-negative PCNSL showed a mild-to-moderate increase in $\mathrm{rCBV}_{\text {max }}$ and $\mathrm{rCBV}_{\mathrm{r}}$ (Table 2). In 22 patients (6 EBV-positive and $16 \mathrm{EBV}$-negative) with interpretable spectra, the Cho peak was increased and the NAA peak was decreased, resulting in an increased Cho/NAA ratio (Table 2). $\mathrm{ADC}_{\text {solid, }}$, tumor perfusion, and the Cho/NAA ratio were not significantly different between EBV-positive and -negative patients with $P>.05$ for all comparisons.

\section{Follow-Up Results of Patients with EBV-Positive PCNSL}

The mean follow-up period of the 10 patients with EBV-positive PCNSL was 17.3 months (range, 3-50 months). Among these 10 

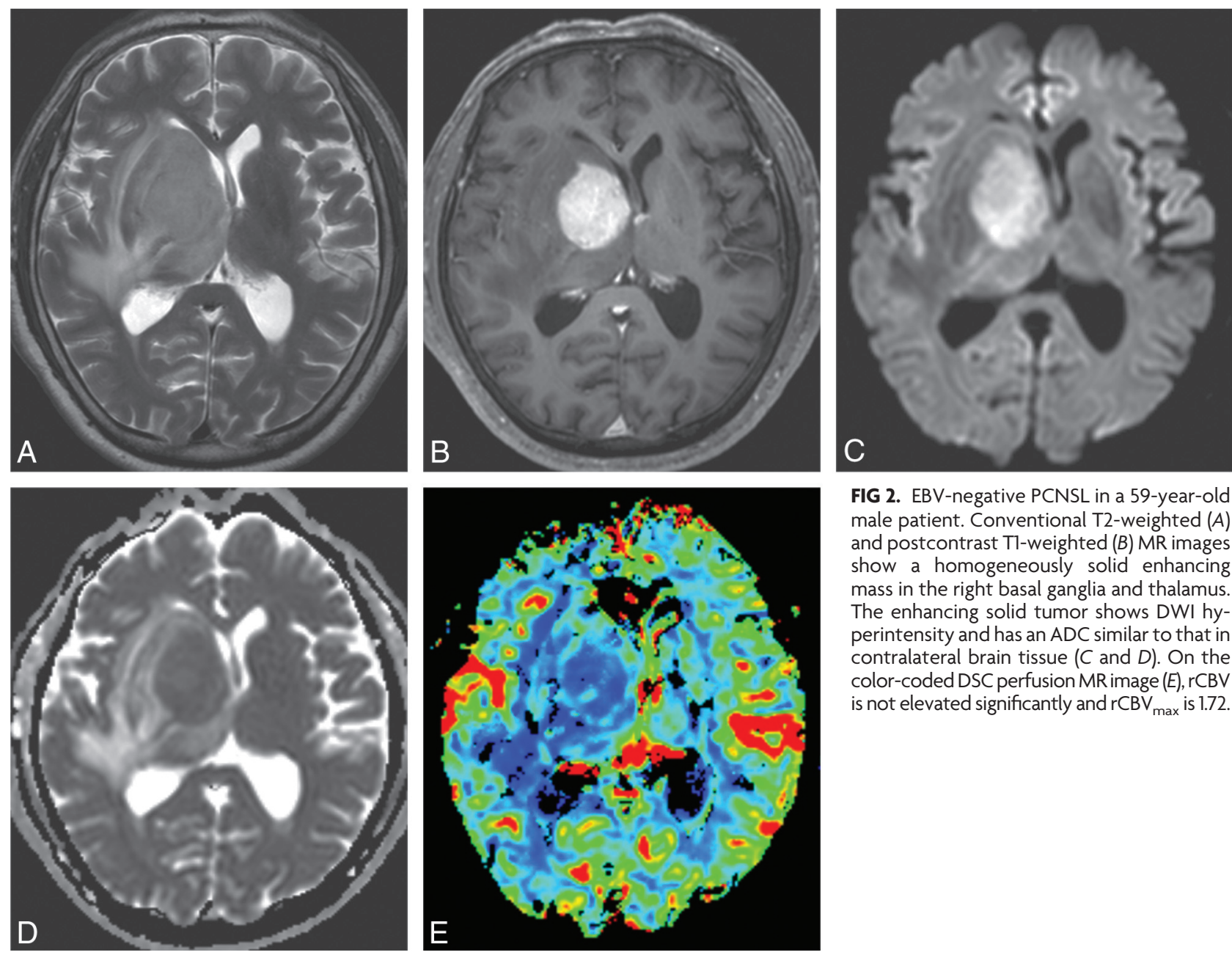

FIG 2. EBV-negative PCNSL in a 59-year-old male patient. Conventional T2-weighted $(A)$ and postcontrast T1-weighted (B) MR images show a homogeneously solid enhancing mass in the right basal ganglia and thalamus. The enhancing solid tumor shows DWI hyperintensity and has an $A D C$ similar to that in contralateral brain tissue $(C$ and $D)$. On the color-coded DSC perfusion MR image (E), rCBV is not elevated significantly and $\mathrm{rCBV}_{\max }$ is 1.72 .

patients, 1 was lost to follow-up and complete remission was achieved in 3 (30\%) and partial treatment response, in 5 (50\%), whereas there was disease progression in 1 patient (10\%) (Table 1).

\section{DISCUSSION}

We compared the MR imaging features of the 10 patients without AIDS with EBV-positive PCNSL with those of the 45 patients without AIDS with EBV-negative PCNSL. The imaging features in these EBV-positive patients were atypical of non-AIDS PCNSL, more closely resembling glioblastoma and including lesions with necrosis, hemorrhage, and irregular or peripheral enhancement. In contrast, most of the EBV-negative cases of PCNSL presented as single or multiple relatively homogeneously enhanced mass lesions, findings typical of non-AIDS PCNSL. ${ }^{12,13}$

On conventional MR imaging, PCNSL in an immunocompetent patient usually presents as a solitary homogeneously enhancing parenchymal mass. Multiple lesions are not uncommon, being reported in $20 \%-40 \%$ of immunocompetent patients with PCNSL and with ringlike enhancement reported in up to $13 \%$ of these patients. ${ }^{14}$ In contrast, irregular, peripheral, and ringlike enhancement is frequently observed in patients with AIDS with PCNSL due to necrosis and is reported in up to $75 \%$ of these patients. ${ }^{12,13}$ On conventional MR imaging, EBV-positive PCNSL in patients without AIDS and AIDS-related PCNSL share several atypical features, as seen in our study. For example, hemorrhage and necrosis were frequent (78\% and $89 \%$, respectively), thus resulting in irregular or peripheral ringlike enhancement. In our study, although the incidence of tumor necrosis was statistically significant between the EBV-positive and EBV-negative groups, we identified findings of tumor necrosis in 8 patients with EBV-negative PCNSL. Therefore, additional studies with a larger patient population should be performed to clarify the true incidence of tumor necrosis in patients with EBV-negative PCNSL. On advanced imaging, in agreement with previous findings, we observed that the $\mathrm{ADC}$ values in solid tumor areas were relatively low $^{14-16}$ and the mean regional CBV ratios were not as high in both EBV-positive and EBV-negative PCNSL as in highgrade gliomas. ${ }^{17}$

There have been a few reports regarding the association of EBV and PCNSL. Although the relevance of EBV in PCNSL to tumorigenesis in immunocompetent hosts remains unclear, EBV sequences were observed in 9 lymphomas (100\%) in immunocompromised patients but in only 2 of 17 (11.8\%) lymphomas in immunocompetent patients. ${ }^{3}$ The association between EBV and PCNSL is not restricted to patients with severe primary immunodeficiency. ${ }^{1}$ For example, EBV-positive PCNSL was observed in 6 patients without predisposing immunodeficiencies. ${ }^{14}$ EBV-positive PCNSL in patients without AIDS tends to develop more frequently in patients 60 years of age and older and has various clinical courses. ${ }^{18} \mathrm{EBV}$ positive PCNSL has a male predominance and can occur in patients with classic immunosuppressed conditions such as human immunodeficiency virus infection, chronic alcoholism, and several collagen 
vascular diseases. ${ }^{3}$ The recent finding of senile EBV-associated, B-cell lymphoproliferative disorder in 22 Japanese patients 60 years of age or older and without predisposing immunodeficiencies suggested that this condition is related to immunologic deterioration associated with the aging process. ${ }^{4,7}$

In our study, we also found that EBV-positive immunocompetent PCNSL occurred primarily in elderly patients except for 2 who were younger than 50 years of age and without predisposing immunodeficiencies, thus suggesting a relationship between this disease and aging-associated immunologic deterioration. On the other hand, as shown by previous studies, the mean age of study patients with PCNSL without known EBV positivity was also $>55$ years. ${ }^{19,20}$ In our experience with a small study population, we did not find an age difference between EBV-positive and EBV-negative patient groups. Further studies with a larger patient population will be needed to verify this demographic finding.

A few published studies support a possible prognostic relationship between the EBV tumoral status and diffuse large B-cell lymphoma. One study revealed that patients with EBV-positive diffuse large B-cell lymphoma had a shorter survival rate than patients with EBV-negative diffuse large B-cell lymphoma. ${ }^{7}$ In our experience, disease progression was observed in 1 patient with EBV-positive PCNSL. However, we did not perform a formal survival analysis because we had only a small patient population and relatively shortterm follow-up results.

Our study had several limitations, including its retrospective design, its performance at a single tertiary hospital, and the small patient population, thus suggesting the possibility of a selection bias. Our comparisons of EBV-positive and -negative patients were limited because they were not cross-matched by age and sex. Finally, the follow-up period was not sufficient to assess either the treatment response or the prognosis. However, because the incidence of PCNSL is rare and the concept of age-related EBV-associated lymphoproliferative disorder was only recently proposed, several of these limitations were unavoidable. Our findings are still important because they show under-recognized MR imaging features of EBV-positive PCNSL and identify several atypical features resembling AIDS-related PCNSL and glioblastoma.

\section{CONCLUSIONS}

Our small patient population study suggests that EBV-positive PCNSL in patients without AIDS showed different imaging features from those of EBV-negative PCNSL. The former usually showed extensive necrosis and hemorrhage, thus resembling the features of glioblastomas. Because the association of PCNSL with EBV may affect a patient's prognosis, patients with PCNSL showing atypical imaging features should be examined for EBV.

\section{REFERENCES}

1. Rouah E, Rogers BB, Wilson DR, et al. Demonstration of EpsteinBarr virus in primary central nervous system lymphomas by the polymerase chain reaction and in situ hybridization. Hum Pathol 1990;21:545-50

2. List AF, Greer JP, Cousar JP, et al. Primary brain lymphomas in the immunocompetent host: relation to Epstein-Barr virus. Mod Pathol 1990;3:609-12

3. Kleinschmidt-DeMasters BK, Damek DM, Lillehei KO, et al. Epstein Barr virus-associated primary CNS lymphomas in elderly patients on immunosuppressive medications. J Neuropathol Exp Neurol 2008;67:1103-11

4. Oyama T, Ichimura K, Suzuki R, et al. Senile EBV+ B-cell lymphoproliferative disorders: A clinicopathologic study of 22 patients. Am J Surg Pathol 2003;27:16-26

5. Nakamura S, Jaffe ES, Swerdlow SH. EBV positive diffuse large Bcell lymphoma of the elderly. In: Swerdlow SH, Campo E, Harris NL, et al, eds. WHO Classification of Tumours of Haematopoietic and Lymphoid Tissues. Lyon, France: IARS Press; 2008:243-44

6. Shimoyama Y, Asano N, Kojima M, et al. Age-related EBV-associated B-cell lymphoproliferative disorders: diagnostic approach to a newly recognized clinicopathological entity. Pathol Int 2009;59:835-43

7. Oyama T, Yamamoto K, Asano N, et al. Age-related EBV associated B-cell lymphoproliferative disorders constitute a distinct clinicopathologic group: a study of 96 patients. Clin Cancer Res 2007;13:5124-32

8. Asano N, Yamamoto K, Tamaru JI, et al. Age-related EBV associated B-cell lymphoproliferative disorders: comparison with EBV-positive classical Hodgkin lymphoma in elderly patients. Blood 2009; 113:2629-36

9. Park S, Lee J, Ko YH, et al. The impact of the Epstein-Barr virus status on clinical outcome in diffuse large B cell lymphoma. Blood 2007;110:972-78

10. Emblem KE, Nedregaard B, Nome T, et al. Glioma grading by using histogram analysis of blood volume heterogeneity from MR-derived cerebral blood volume maps. Radiology 2008;247:808-17

11. Law M, Young R, Babb J, et al. Histogram analysis versus region of interest analysis of dynamic susceptibility contrast perfusion MR imaging data in the grading of cerebral gliomas. AJNR Am J Neuroradiol 2007;28:761-66

12. Thurnher MM, Rieger A, Kleibl-Popov C, et al. Primary central nervous system lymphoma in AIDS: a wider spectrum of CT and MRI findings. Neuroradiology 2001;43:29-35

13. Haldorsen IS, Kråkenes J, Krossnes BK, et al. CT and MR imaging features of primary central nervous system lymphoma in Norway, 1989-2003. AJNR Am J Neuroradiol 2009;30:744-51

14. Guo AC, Cummings DJ, Dash RC, et al. Lymphomas and high-grade astrocytomas: comparison of water diffusibility and histologic characteristics. Radiology 2002;224:177-83

15. Toh CH, Castillo M, Wong AM-C, et al. Primary cerebral lymphoma and glioblastoma multiforme: differences in diffusion characteristics evaluated with diffusion tensor imaging. AJNR Am J Neuroradiol 2008;29:471-75

16. Krabbe $\mathrm{K}$, Gideon $\mathrm{P}$, Wagn $\mathrm{P}$, et al. MR diffusion imaging of human intracranial tumours. Neuroradiology 1997;39:483-89

17. Calli $\mathrm{C}$, Kitis $\mathrm{O}$, Yunten $\mathrm{N}$, et al. Perfusion and diffusion MR imaging in enhancing malignant cerebral tumors. Eur J Radiol 2006;58:394-403

18. Sugita Y, Terasaki M, Niino D, et al. Epstein-Barr virus- associated primary central nervous system lymphomas in immunocompetent elderly patients: analysis for latent membrane protein-1 oncogene deletion and EBNA-2 strain typing. J Neurooncol 2010;100:271-79

19. Barajas RF Jr., Rubenstein JL, Chang JS, et al. Diffusion-weighted MR imaging derived apparent diffusion coefficient is predictive of clinical outcome in primary central nervous system lymphoma. AJNR Am J Neuroradiol 2010;31:60-66

20. Wieduwilt MJ, Valles F, Issa $S$, et al. Immunochemotherapy with intensive consolidation for primary central nervous system lymphoma: a pilot study and prognostic assessment by diffusionweighted MRI. Clin Cancer Res 2012;18:1146-55 